\title{
2 Immune Cells vs. Cancer Cells: A Microscopic Energy Battle
}

3

$4 \quad$ Farzad Taghizadeh-Hesary ${ }^{\mathrm{I}, 2, \uparrow}$, Hassan Akbari ${ }^{3,4, \dagger}$, Moslem Bahadori $^{5}$

6

7

1. Department of Radiation Oncology, Iran University of Medical Sciences, Tehran, Iran. Hospital, Iran University of Medical Sciences, Tehran, Iran

3. Department of Pathology, Shahid Beheshti University of Medical Sciences, Tehran, Iran.

4. Traditional Medicine School, Tehran University of Medical Sciences, Tehran, Iran.

\section{Corresponding authors:}

1. Farzad Taghizadeh-Hesary, M.D.

Primary E-mail: f_taghizadeh@sbmu.ac.ir

Secondary E-mail: Farzadth89@gmail.com

Cell phone: +989126086713

Tel: +9826651517

Fax: +9826651517

ORCiD: 0000-0002-6195-2203

\section{Hassan Akbari, M.D.}

E-mail address: hmhakbari@yahoo.com

Cell phone: +989121785345

Traditional Medicine School, Tehran University of Medical Sciences, Tehran, Iran.

2. ENT and Head \& Neck Research Center and Department, The Five Senses Health Institute, Hazrat Rasoul Akram

5. Professor emeritus, Faculty of Medicine, Tehran University of Medical Sciences, Tehran, Iran.

Assistant Professor, Department of Oncology, Iran University of Medical Sciences, Tehran, Iran

Associate Professor, Department of Pathology, Shahid Beheshti University of Medical Sciences, Tehran, Iran. 


\section{Immune Cells vs. Cancer Cells: A Microscopic Energy Battle}

45 Abstract

46 Like living organisms, cancer cells require energy to survive and interact with their environment.

47 Recently, investigators demonstrated that cancer cells can hijack mitochondria from immune cells.

48 This behavior sheds light on a pivotal piece in the puzzle of cancer, the 'dependence'. This article

49 illustrates how new, functional mitochondria help cancer cells to survive in the harsh tumor

50 microenvironment, evade immune cells, and improve their malignancy. Finally, we will discuss how

51 blocking the routes supplying energy for cancer cells can improve the treatment outcomes of

52 radiotherapy, chemotherapy, and immunotherapy. This article provides a new theory in oncology,

53 the 'energy battle' between cancer and immune cells. It alludes each party with a higher energy level

54 can be the winner. This theory explains cancer biogenesis and provides novel insights to improve

55 treatment outcomes.

56 Keywords: ATP; Cancer cell; Mitochondria; T cell

57

1. Introduction

All living organisms require energy for their maintenance, growth, repopulation, and appropriate response to external stimuli. Some organisms are self-sufficient ('autotrophs') and acquire energy from sunlight or chemicals. The remaining organisms ('heterotrophs') rely on autotrophs to secure energy ${ }^{[1]}$. A recent in vitro experiment from the United States demonstrated that cancer cells are dependent on normal cells for their living and function. In Nov 2021, Saha et al. demonstrated that cancer cells can hijack mitochondria (the cell's energy factories) from immune cells via nanoscale tube-like structures ${ }^{[2]}$. Besides providing energy, mitochondria are essential mediators for cancer cells' survival and evolution. In the following section, we describe how new, functional mitochondria are vital for evolving cancer.

2. Mitochondria's Benefits for Cancer Cells

Mitochondria bring four crucial benefits for cancer cells (Figure 1);

71

\subsection{Surviving in the Harsh Tumor Microenvironment}

Cancer cells can survive in the harsh tumor microenvironment (TME) by (1) metabolic

74 switch to aerobic glycolysis (Warburg effect), (2) enhanced antioxidant capacity, (3) protective cell cycle arrest ('quiescence' or 'dormancy'), and (4) autophagy ${ }^{[3]}$. Mitochondria are involved in the aforementioned four strategies. Hexokinase (HK) is the rate-limiting enzyme of glycolysis, which catalyzes the phosphorylation of glucose to glucose-6P. Functional mitochondria enable cancer cells 
79

80

81

82

83

84

85

86

87

88

89

90

91

92

93

94

95

96

97

98

99

100

101

102

103

104

105

106

107

108

109

110

111

112

113

114

115

116

117

the negative-feedback effect of downstream glucose-6P ${ }^{[4]}$. The sustained glycolytic pathway provides three benefits for cancer cells: (1) aerobic glycolysis can satisfy the anabolic demands of cancer cells by providing lipids, proteins, and nucleotides ${ }^{[5]}$; (2) the pyruvates (interim products of aerobic glycolysis) can serve as an antioxidant and neutralizes the intracellular reactive oxygen species (ROS)_as a byproduct of cellular metabolism ${ }^{[6]}$; and (3) normoxic cancer cells can utilize lactate (final products of glycolysis) as an energy source ('metabolic symbiosis') ${ }^{[5]}$. Emerging evidence has put forward the mitochondria reaction to hypoxia in dormant cancer cells. In an in vitro model of dormant breast cancer, chronic hypoxia led to a marked increase in mitochondria number and mitochondrial ROS (mtROS)_denoting mitochondria metabolism ${ }^{[7]}$. In addition, mitochondria hijacking enables cancer cells to replace the old, defective mitochondria (degraded by mitophagy) with the new, functional mitochondria from immune cells to reply to the mitochondria demands ${ }^{[2]}$.

\subsection{Immune evasion}

Mitochondrial hijacking also enables immune evasion by depleting the energy of immune cells. Ample evidence has revealed that $\mathrm{T}$ cells (as the lead of immune response to cancer) require energy for the proper activation against cancer cells ${ }^{[8]}$. This mechanism is next to the formerly recognized immune evasions mechanisms of cancer cells, including expressing immune checkpoint inhibitors, drawing tumor-derived regulatory $\mathrm{T}$ cells and myeloid-derived suppressor cells (MDSCs) into TME, defective antigen presentation, and releasing immune-suppressive mediators ${ }^{[9]}$. In addition, the new functional mitochondria enable cancer cells to run more aerobic glycolysis. The glycolysis upregulation leads to TME acidosis by disposing of lactate-as the end product of aerobic glycolysis - in the extracellular milieu. In low-pH TME, T cells lose their function and enter a state of anergy followed by apoptosis. Moreover, the activated glycolysis leads to enhanced expression of glucose transporters (such as GLUT-1) and glycolytic enzymes in cancer cells. This process is mediated by lactate-induced hypoxia-inducible factor- $1 \alpha$ (HIF-1 $\alpha)$ overexpression, which in turn upregulates the GLUT-1 ${ }^{[10-12]}$. This process makes the glucose out of the reach of $\mathrm{T}$ cells and further impedes the appropriate function of immune cells.

\subsection{Upgrading Malignancy}

Mitochondria generate $90 \%$ of the total cellular ROS volume ${ }^{[13]}$. Cancer cells with more functional mitochondria have an elevated 'ROS balance'. In other words, they can generate more mtROS on one hand, and better remove the generated ROS on the other hand. The former gives rise to genomic instability, cell cycle checkpoint evasion, and enhances the ability to metastasize (by driving epithelial-mesenchymal transition), and the latter impedes self-damage of oxidative stress to mitochondrial and cellular nucleic acid, proteins, and lipids ${ }^{[14]}$.

\subsection{Resistance to Treatment}

Most chemotherapeutics trigger cell death through oxidative stress. This is mediated by damage to cancer cell components and promoting apoptosis ${ }^{[15]}$. Ionizing radiation can damage cancer 
118

cells by direct damage to DNA or dominantly through ROS generation and indirect damages to cellular or mitochondrial components ${ }^{[16]}$. Mitochondria protect cancer cells from chemotherapy and radiotherapy by scavenging the generated ROS. Besides, mitochondria can give rise to multidrug resistance by providing enough ATP molecules for the ATP-dependent multidrug efflux pumps ${ }^{[17]}$. In addition to radiotherapy and chemotherapy, mitochondria can enhance the resistance to immunotherapy. This notion was demonstrated in an in vivo experiment, in which blocking the mitochondria trafficking from $T$ cells to cancer cells improved the efficacy of anti-programmed cell death protein-1 (anti-PD-1) immunotherapy ${ }^{[2]}$.

\section{Discussion}

In this article, we demonstrated the vital role of mitochondria in cancer cells' survival, malignancy, and the confrontation with immune cells. In the struggle between immune and cancer cells, each party with a higher energy level can win the battle. More functional mitochondria empower the cancer cells and enable them to overcome their opponent, the immune cells.

As alluded to above, more functional mitochondria can better scavenge the ROSs and increase the resistance threshold of cancer cells to radiotherapy and chemotherapy. Moreover, mitochondria hijacking from immune cells upgrades the cancer cells' resistance to anti-PD-1 antibodies ${ }^{[2]}$. This finding supports the hypothesis that $T$ cells' mitochondria status determines response to anti-PD-1 immunotherapy ${ }^{[18]}$. In Jan 2021, Akbari and Taghizadeh-Hesary et al. demonstrated that T cells' mitochondrial activation can improve the response to anti-PD-1 antibodies by improving recognition (through PD-1 downregulation on $\mathrm{T}$ cells) and providing energy for long-term $\mathrm{T}$ cell activation.

These strategic findings can introduce a new, potential theory in oncology, the 'energy battle'. In this theory, shifting the energy balance toward the immune cells can improve the radiotherapy, chemotherapy, and immunotherapy outcomes. Theoretically, leveling up the immune cells (against cancer cells) can potentially serve as monotherapy. Immune cells with stronger mitochondria are more efficient in all phases of cancer cell recognition (through PD-1 downregulation), activation, proliferation, migration, and cancer cell killing ${ }^{[18-20]}$. All these phases are ATP-dependent ${ }^{[8,18]}$. On the other hand, cancer cells with weaker mitochondria cannot tolerate the bulk of ROSs generated during radiotherapy and chemotherapy. Shifting the energy balance toward the immune cells can be achieved by improving $\mathrm{T}$ cells' mitochondria in quantity and quality. For the primer, the $\mathrm{T}$ cells' mitochondria numbers can be saved by blocking mitochondria hijacking ${ }^{[2]}$. The mitochondria quality can increase by two strategies; (1) improving the lifestyle by regular exercise ${ }^{[21]}$, low-SDA (specific dynamic action) diet ${ }^{[22]}$, good sleep ${ }^{[23]}$, healthy weight ${ }^{[24]}$, and smoking cessation ${ }^{[25]}$; and (2) mitochondria boosting agents [e.g., activators of adenosine monophosphate-activated protein kinase (AMPK), mammalian target of rapamycin (mTOR), and peroxisome proliferator-activated receptor-gamma coactivator 1 -alpha $(\mathrm{PGC}-1 \alpha)]^{[26]}$. 


\section{Conclusions}

Hitherto, scientists believed that cancer is an autonomous organism that does not obey the general body regulations ${ }^{[27]}$. This notion seems to be true; however, it is not the whole story. The recent finding of mitochondria hijacking from immune cells revealed another behavior of cancer cells, the reliance on normal cells for survival and function. This feature seems to be cancer's Achilles' heel, and the human being can overcome cancer by targeting this - and the possible other - route for energy supply. Further studies are warranted to examine this theory.

\section{Declarations}

Funding: None

Conflicts of interest: The authors declare that they have no competing interests.

Availability of data and material: Not applicable

Acknowledgement: None

\section{References}

1. Halvorson $\mathrm{HM}$, Wyatt $\mathrm{KH}$, Kuehn KA. Ecological significance of autotroph-heterotroph microbial interactions in freshwaters. Freshwater Biology. 2020;65(7):1183-8.

2. Saha T, Dash C, Jayabalan R, Khiste S, Kulkarni A, Kurmi K, et al. Intercellular nanotubes mediate mitochondrial trafficking between cancer and immune cells. Nature nanotechnology. 2021:1-9.

3. Akbari H, Taghizadeh-Hesary F, Heike $\mathrm{Y}$, Bahadori M. Cell energy: A new hypothesis in decoding cancer evolution. Archives of Iranian Medicine (AIM). 2019;22(12).

4. Pastorino JG, Hoek JB. Hexokinase II: the integration of energy metabolism and control of apoptosis. Current medicinal chemistry. 2003;10(16):1535-51.

5. Vaupel P, Multhoff G. Revisiting the Warburg effect: historical dogma versus current understanding. The Journal of Physiology. 2021;599(6):1745-57.

6. Paredes F, Williams HC, San Martin A. Metabolic adaptation in hypoxia and cancer. Cancer letters. 2021.

7. Carcereri de Prati A, Butturini E, Rigo A, Oppici E, Rossin M, Boriero D, et al. Metastatic breast cancer cells enter into dormant state and express cancer stem cells phenotype under chronic hypoxia. Journal of cellular biochemistry. 2017;118(10):3237-48.

8. Herbel C, Patsoukis N, Bardhan K, Seth P, Weaver JD, Boussiotis VA. Clinical significance of T cell metabolic reprogramming in cancer. Clinical and translational medicine. 2016;5(1):1-23.

9. Vinay DS, Ryan EP, Pawelec G, Talib WH, Stagg J, Elkord E, et al., editors. Immune evasion in cancer: Mechanistic basis and therapeutic strategies. Seminars in cancer biology; 2015: Elsevier. 
10. Bao X, Zhang J, Huang G, Yan J, Xu C, Dou Z, et al. The crosstalk between HIFs and mitochondrial dysfunctions in cancer development. Cell Death \& Disease. 2021;12(2):1-13.

11. Yu X-J, Song J-C, Du J, Shi Y-Q, Liu Y-X, Shen Y. GLUT-1 and its regulating factor HIF-1 $\alpha$ expression in epithelial ovarian tumors: GLUT-1 is associated with molecular typing and grade of epithelial ovarian cancer. Int J Clin Exp Pathol [Internet]. 2017;10(4):4479-87.

12. Huber V, Camisaschi C, Berzi A, Ferro S, Lugini L, Triulzi T, et al., editors. Cancer acidity: An ultimate frontier of tumor immune escape and a novel target of immunomodulation. Seminars in cancer biology; 2017: Elsevier.

13. Wang $\mathrm{H}$, Jiang $\mathrm{H}$, Van De Gucht $\mathrm{M}$, De Ridder M. Hypoxic radioresistance: can ROS be the key to overcome it? Cancers. 2019;11(1):112.

14. Dunn JD, Alvarez LA, Zhang X, Soldati T. Reactive oxygen species and mitochondria: A nexus of cellular homeostasis. Redox biology. 2015;6:472-85.

15. Yang $\mathrm{H}$, Villani RM, Wang $\mathrm{H}$, Simpson MJ, Roberts MS, Tang $\mathrm{M}$, et al. The role of cellular reactive oxygen species in cancer chemotherapy. Journal of Experimental \& Clinical Cancer Research. 2018;37(1):1-10.

16. Desouky O, Ding N, Zhou G. Targeted and non-targeted effects of ionizing radiation. Journal of Radiation Research and Applied Sciences. 2015;8(2):247-54.

17. Perillo B, Di Donato M, Pezone A, Di Zazzo E, Giovannelli P, Galasso G, et al. ROS in cancer therapy: The bright side of the moon. Experimental \& Molecular Medicine. 2020;52(2):192-203.

18. Akbari H, Taghizadeh-Hesary F, Bahadori M. Mitochondria determine response to antiprogrammed cell death protein-1 (anti-PD-1) immunotherapy: An evidence-based hypothesis. Mitochondrion. 2022;62:151-8.

19. Desdín-Micó G, Soto-Heredero G, Mittelbrunn M. Mitochondrial activity in T cells. Mitochondrion. 2018;41:51-7.

20. Surace L, Doisne J-M, Escoll P, Marie S, Dardalhon V, Croft C, et al. Polarized mitochondria as guardians of NK cell fitness. Blood advances. 2021;5(1):26-38.

21. Memme JM, Erlich AT, Phukan G, Hood DA. Exercise and mitochondrial health. The Journal of physiology. 2021;599(3):803-17.

22. Luoma RL, Butler MW, Stahlschmidt ZR. Plasticity of immunity in response to eating. Journal of Experimental Biology. 2016;219(13):1965-8.

23. Rodrigues NR, Macedo GE, Martins IK, Gomes KK, de Carvalho NR, Posser T, et al. Short-term sleep deprivation with exposure to nocturnal light alters mitochondrial bioenergetics in Drosophila. Free Radical Biology and Medicine. 2018;120:395-406.

24. de Mello AH, Costa AB, Engel JDG, Rezin GT. Mitochondrial dysfunction in obesity. Life sciences. 2018;192:26-32.

25. Malińska D, Więckowski MR, Michalska B, Drabik K, Prill M, Patalas-Krawczyk P, et al. Mitochondria as a possible target for nicotine action. Journal of bioenergetics and biomembranes. 2019;51(4):259-76.

26. Chamoto K, Chowdhury PS, Kumar A, Sonomura K, Matsuda F, Fagarasan S, et al. Mitochondrial activation chemicals synergize with surface receptor PD-1 blockade for T cell-dependent antitumor activity. Proceedings of the National Academy of Sciences. 2017;114(5):E761-E70.

27. Duesberg P, Mandrioli D, McCormack A, Nicholson JM. Is carcinogenesis a form of speciation? Cell cycle. 2011;10(13):2100-14. 


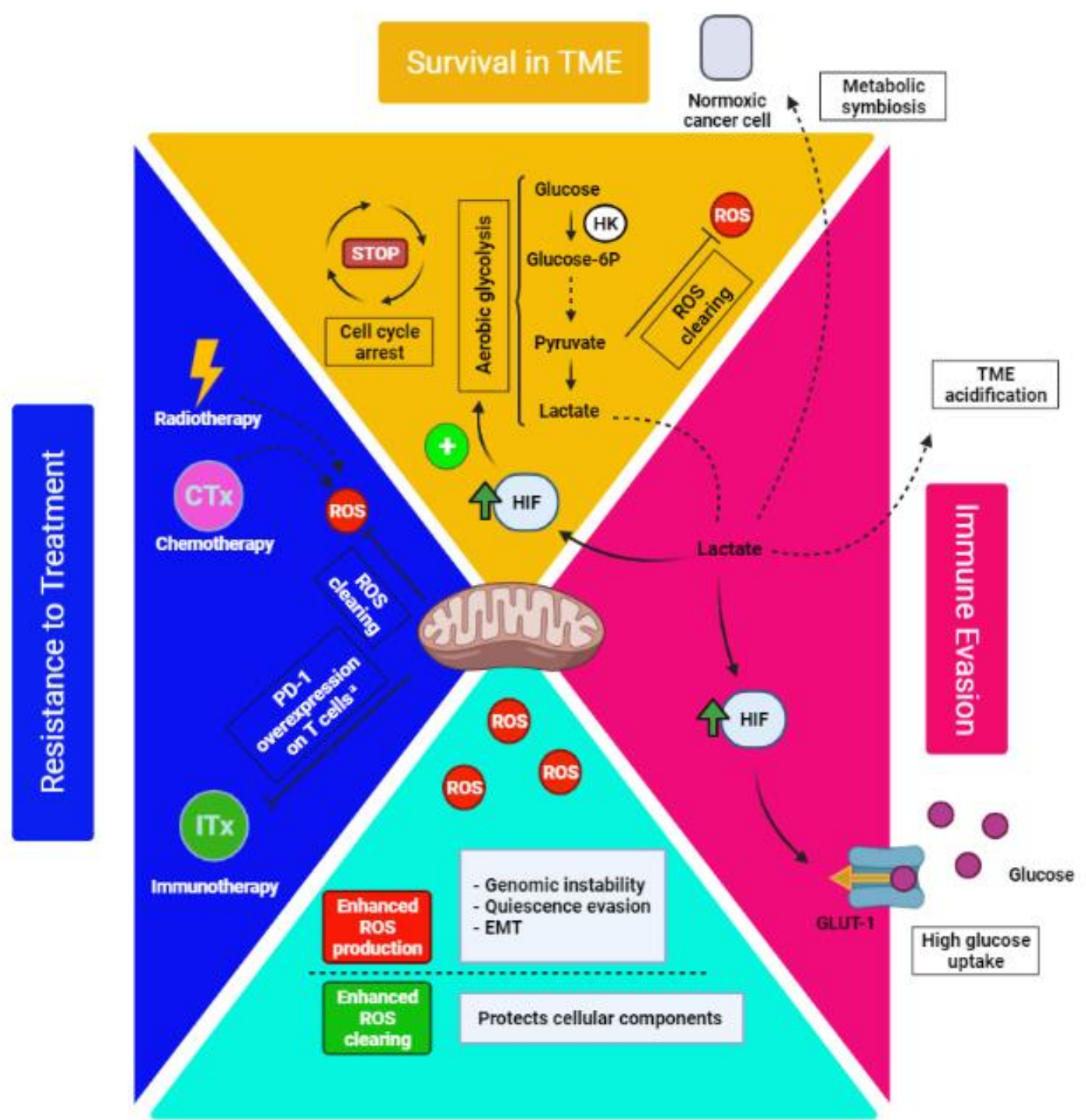

Malignancy Upgrading

242 Figure 1. The pivotal role of mitochondria in cancer survival, immune evasion, malignancy, and resistance to treatments.

243 Abbreviations: EMT, epithelial-mesenchymal transition; GLUT-I, Glucose transporter-1; HIF, hypoxia-inducible

244 factor; HK, hexokinase; PD-1, programmed cell death protein-1; ROS, reactive oxygen species; TME, tumor 245 microenvironment. (Created with BioRender.com) 